\title{
Estudio de remoción de arsénico en agua potable a nivel domiciliar mediante oxidación solar y coagulación-floculación
}

\author{
Study of arsenic removal in drinking water \\ at household level using solar oxidation and \\ coagulation-flocculation techniques
}

\section{Paola Rojas-Chaves ${ }^{1}$, María J. Vargas-Benavides ${ }^{2}$, Andrés Araya- Obando ${ }^{3}$, Johnny Valverde-Cerdas ${ }^{4}$, Luis G. Romero-Esquivel ${ }^{5}$}

Fecha de recepción: 23 de marzo del 2015

Fecha de aprobación: 11 de junio del 2015

Rojas-Chaves, P; Vargas-Benavides, M; Araya-Obando, A; Valverde-Cerdas, J; Romero-Esquivel, L. G. Estudio de remoción de arsénico en agua potable a nivel domiciliar mediante oxidación solar y coagulación-floculación. Tecnología en Marcha. Vol. 28, № 4, Octubre-Diciembre. Pág 54-65.

1 Graduada de Ingeniería Ambiental. Instituto Tecnológico de Costa Rica Teléfono: (506)89905193, Cartago, Costa Rica. Correo electrónico: projas1208@gmail.com

2 Estudiante de Ingeniería Ambiental. Instituto Tecnológico de Costa Rica. Teléfono: (506)8876-0210, Cartago, Costa Rica. Correo electrónico: mariajvargas@gmail.com

3 Investigador de la Escuela de Ingeniería en Construcción. Instituto Tecnológico de Costa Rica. Teléfono: (506)2550-2311, Cartago, Costa Rica. Correo electrónico: jobando@itcr.ac.cr

4 Investigador. Escuela de Química. Instituto Tecnológico de Costa Rica. Teléfono: (506)25502734, Cartago, Costa Rica. Correo electrónico: jovalverde@itcr.ac.cr

5 Investigador. Escuela de Química. Instituto Tecnológico de Costa Rica. Teléfono: (506)25502304, Cartago, Costa Rica. Correo electrónico: Iromero@itcr.ac.cr 
Tecnología en Marcha,

Vol. 28, N. ${ }^{\circ} 4$, Octubre-Diciembre 201555

\section{Palabras clave}

Agua de consumo humano; remoción de arsénico; oxidación solar; floculación; coagulación.

\section{Resumen}

En Costa Rica, a pesar de que se han hecho esfuerzos por abastecer de agua segura a las comunidades afectadas por la presencia de arsénico en el líquido, una parte de la población aún se encuentra expuesta, posiblemente debido a que no están conectadas a una red de acueductos. Es por ello que en este estudio se presentan dos soluciones a nivel domiciliar para eliminar el arsénico: una por oxidación solar (SORAS) y otra por un proceso de coagulaciónfloculación. En ambos casos, el arsénico es removido por adsorción o coprecipitación sobre los óxidos hidróxidos de hierro (III) formados.

En el sistema SORAS se encontró que para reducir la concentración de arsénico de 200 $\mathrm{\mu g} / \mathrm{L}$ a menos de la norma $(10 \mu \mathrm{g} / \mathrm{L})$, se necesitan entre $2 \mathrm{~h}$ y $4 \mathrm{~h}$ de exposición en un día soleado o nublado, respectivamente. En el sistema de coagulación-floculación, las condiciones óptimas para obtener niveles de arsénico por debajo de la norma fueron las siguientes: con floculante sintético, el pH fue 6 con $1 \mathrm{mg} / \mathrm{L}$ de FK-930-S (polímero de cloruro de polidialil-dimetilamonio) con dosis de $\mathrm{FeCl}_{3} 12 \mathrm{mg} / \mathrm{L}$; con floculante natural, el pH fue 6 con $250 \mathrm{mg} / \mathrm{L}$ de mozote (Triumfetta semitriloba), con dosis de $\mathrm{FeCl}_{3}$ de $14 \mathrm{mg} / \mathrm{L}$. Un prototipo consistente en un recipiente de coagulación-floculación y otro de filtración en arena fue capaz de tratar $15 \mathrm{~L}$ de agua sintética con $200 \mu \mathrm{g} / \mathrm{L}$ As y agua natural con 10 y $50 \mu \mathrm{g} / \mathrm{L}$. A pesar de estos resultados, es necesario evaluar ambas técnicas en las comunidades, incluyendo aspectos de capacitación y aceptación.

\section{Keywords}

Drinking water; arsenic removal; solar oxidation; flocculation; coagulation.

\section{Abstract}

In Costa Rica, even though there have been efforts to provide safe water to communities affected by the presence of arsenic, there is a part of the population still exposed because might not be connected to a network of aqueducts. Two solutions household level use for As reduction are presented: one is the solar radiation oxidation (SORAS) and second one is the flocculation - coagulation process. Both solutions showed reduced As concentration due to adsorption or co-precipitation on the iron (III) oxide hydroxides. The SORAS system needed $2 \mathrm{~h}$ and $4 \mathrm{~h}$ of exposure in a sunny or cloudy day respectively to reduce the arsenic concentration from 200 $\mu \mathrm{g} /$ $\mathrm{L}$ to less than the standard $(10 \mathrm{ug} / \mathrm{L}$ ). For coagulation - flocculation system, best conditions were achieved at pH 6 with synthetic flocculant FK-930-S (polymer polydiallyl-dimethylammonium chloride) at $1 \mathrm{mg} / \mathrm{L}$ dose and $\mathrm{FeCl}_{3} 12 \mathrm{mg} / \mathrm{L}$ dose; for natural flocculant it was achieved at $\mathrm{pH}$ 6 with mozote (Triumfetta semitriloba) at $250 \mathrm{mg} / \mathrm{L}$ dose, and $\mathrm{FeCl}_{3} 14 \mathrm{mg} / \mathrm{L}$ dose. A prototype consisting in a coagulation-flocculation container and a sand filtration step was able to treat 15 liters of synthetic water with 200 $\mathrm{\mu g} / \mathrm{L}$ As and natural water with 10 and $50 \mathrm{ug} / \mathrm{L}$. Despite these results, it is necessary to evaluate both techniques in communities, including aspects of training and acceptance. 
Tecnología en Marcha,

56 Vol. 28, N. ${ }^{\circ}$ 4, Octubre-Diciembre 2015

\section{Introducción}

El consumo de agua con arsénico en las fuentes naturales es un problema de salud pública que ha sido asociado a cáncer de piel, riñones, pulmones y vejiga (Bundschuh et al., 2012), entre otras enfermedades. Por tal motivo, la Organización Mundial de la Salud (OMS) y el Ministerio de Salud de Costa Rica limitaron la concentración máxima de arsénico en el agua para consumo humano a 10 mg/L (Ministerio de Salud, 2005; OMS, 2011). Por lo anterior, es necesario buscar fuentes de agua alternativas o brindar un tratamiento para la remoción del arsénico.

Entre las tecnologías para remover arsénico más comunes se incluyen la adsorción, el intercambio iónico, las membranas, la oxidación/precipitación y la coagulación-floculación/ coprecipitación (Mohan y Pittman, 2007). En general, en cada uno de estos procesos se requiere una fase previa de oxidación, ya que la remoción de arsénico en la forma pentavalente es más efectiva que en la trivalente. Los oxidantes más utilizados son el hipoclorito de sodio y el permanganato de potasio (OPS, 2005). Una vez realizada la oxidación, en presencia de hierro en el agua, se produce la precipitación de hidróxidos de hierro (III) en cuya superficie es retenido el arsénico pentavalente.

Un sistema de oxidación/precipitación bastante simple y seguro es la remoción de arsénico por oxidación solar (SORAS), que consiste en colocar el agua contaminada en botellas plásticas transparentes con la adición de jugo de limón y hierro (Litter et al., 2010). Posteriormente, las botellas se exponen a radiación solar para oxidar el arsénico trivalente y formar compuestos de coordinación entre el hierro y citrato mediante un proceso foto-fenton (Litter et al., 2010). El arsénico pentavalente coprecipita con los hidróxidos de hierro formados (Litter et al., 2012)

Además, se puede mencionar el frecuente uso de sulfato de aluminio, cloruro de hierro (III) y sulfato de hierro (III) como coagulantes. Las sales de hierro han demostrado ser más eficientes en la remoción de arsénico que el sulfato de aluminio en un rango de pH más amplio (Jain y Singh, 2012). Por ejemplo, el sistema "Bucket Treatment Unit" (BTU) utiliza dos recipientes; en el primero se realiza un proceso de coagulación-floculación y en el segundo una filtración. Usando cloruro de hierro (III) como coagulante se lograron cambios en la concentración, desde 375-640 $\mu \mathrm{g} / \mathrm{L}$ a valores menores de $20 \mu \mathrm{g} / \mathrm{L}$. El sistema "Fill and Draw Units" es similar al anterior, solo que el proceso se realiza en un solo tanque (Jain y Singh, 2012). Basado en el mismo principio, existe el sistema de la Organización de Salud Pública y Ambiental de Nepal (ENPHO, por sus siglas en inglés), y consiste en un cubo de $20 \mathrm{~L}$ al que se le añade una mezcla de cloruro de hierro (III), carbón en polvo e hipoclorito de sodio, logrando una eficiencia de remoción de alrededor del $91 \%$ con concentraciones desde los 91-274 $\mu \mathrm{g} / \mathrm{L}$ llegando a valores de entre 9 y $22 \mu \mathrm{g} / \mathrm{L}$ de arsénico final (Hwang, 2002). Es factible utilizar un floculante natural como el mozote (Triumfetta semitriloba), ya que acelera el proceso de separación de los floculos (Vargas, 2008) y posterior filtración utilizando un filtro de arena. Este sistema de coagulación-floculacion usando cloruro de hierro (III) y mozote es el que se analiza en el presente estudio.

En Costa Rica, a pesar de que las ASADAS han venido resolviendo el problema mediante sistemas de remoción con óxido de titanio (IV) (Vargas, com. pers., agosto de 2014), existe la posibilidad de encontrar familias que no están conectadas a ellos. Con el objetivo de brindar una alternativa de bajo costo a esta población que no tiene solucionado el problema de exposición al arsénico, se presentan tanto los resultados de ensayos preliminares de remoción por la técnica SORAS modificada como los resultados finales obtenidos usando coagulaciónfloculación con mozote como floculante. 
Tecnología en Marcha,

Vol. 28, N. ${ }^{\circ} 4$, Octubre-Diciembre $2015 \mid 57$

\section{Ensayos con el sistema SORAS modificado}

En estos ensayos se siguió el procedimiento descrito por Litter y colaboradores (2010). En este caso se utilizaron botellas de PET de 600 o $1500 \mathrm{~mL}$ conteniendo agua sintética tipo reto, formulada por la American Science Foundation (NSF) y descrita por Amy y colaboradores (2005) (ver cuadro 1). En dichas botellas se agregó As (V) hasta obtener una concentración de $200 \mu \mathrm{g} / \mathrm{L}$. Posteriormente, se dosificaron 0,6 g/L de hierro cero-valente obtenido de hilos de hierro del utilizado para el lavado de utensilios de cocina o "brillo comercial". Dichas botellas se expusieron a la radiación solar al colocarlas inclinadas a ras del suelo a partir de las 8 am. Posteriormente, se tomó una muestra de agua de una botella diferente cada hora durante las siguientes seis horas. Las muestras de agua se filtraron utilizando papel filtro $<2 \mu \mathrm{m}$ (marca Albet) y acidificaron con ácido nítrico $(1+1)$ para el posterior analisis, al menos en duplicado, de arsénico y hierro. Inmediatamente después de la filtración, se determinó la turbidez y el color del filtrado en algunas muestras. Además, de las botellas mencionadas, se analizó el comportamiento de dos blancos: uno con hierro que no fue expuesto a la radiación solar y otro sin hierro que sí se colocó a la luz solar. En total se realizaron tres experimentos: el primero en un día nublado (A) y el segundo y tercero en días soleados (B y C). La intensidad de la radiación solar fue detectada durante el periodo de los experimentos en una estación meteorológica localizada en el Campus Central del Tecnológico de Costa Rica (TEC) en Cartago.

\section{Ensayos de coagulación-floculación con cloruro de hierro (III) a nivel de laboratorio}

Se utilizó un aparato de prueba de jarras Phipps \& Bird PB-700 con seis jarras, que contenían un litro de agua reto tipo NSF. Mediante las pruebas se definió el pH óptimo, las dosis óptimas de coagulante, floculante y su tiempo óptimo de floculación; además se determinó el tiempo de sedimentación de los flóculos. En cuanto a la determinación de las dosis óptimas, el tiempo de coagulación empleado correspondió a 1 min a 150 rpm y la floculación a 10 min a 20 rpm; variando este último para identificar el tiempo óptimo. Posteriormente, se filtraron las muestras utilizando papel filtro Whatman No. 40. Se utilizó el floculante sintético comercial FK-930-S (polímero de cloruro de polidialil-dimetilamonio) y una disolución de mozote (Triumfetta semitriloba) como floculante natural. Se preparó una disolución madre de 0,1\% v/v de floculante sintético a partir del polímero catiónico 100\% puro. El mozote se preparó a partir de $20 \mathrm{~g}$ cortado en trozos de $2-3 \mathrm{~cm}$, en $100 \mathrm{~mL}$ de agua ultrapura. La disolución se agitó durante 30 min para posteriormente refrigerarse un día antes de su utilización. Para los ensayos, se filtró el jugo de mozote con un filtro de tela de de algodón comercial de los que se usan para chorrear café, obteniéndose una disolución de 200000 mg/L. Todas las pruebas realizadas tuvieron una concentración de arsénico pentavalente cercano a $200 \mu \mathrm{g} / \mathrm{L}$, debido a que este es un valor cercano a uno de los más altos encontrado en la zona afectada, de 0,187 mg/L (187 $\mu \mathrm{g} / \mathrm{L})$ (Astorga, 2013).

\section{Sistema piloto de coagulación-floculación}

Una vez definidas las condiciones apropiadas para la remoción de As, se construyó un sistema con capacidad para producir $15 \mathrm{~L}$ de agua libre de arsénico para su uso a nivel domiciliar. El sistema consistió en un tanque de $20 \mathrm{~L}$ para la coagulación-floculación (tanque para mezcla), que se encuentra en la parte superior (figura 1) y un segundo recipiente también de $20 \mathrm{~L}$ en el que se colocó el medio filtrante. Dicho medio consistió (en orden de superior a inferior): 10 $\mathrm{cm}$ de arena de $0,5 \mathrm{~mm}$ y un lecho de soporte de $2,5 \mathrm{~cm}$ de arena de $1 \mathrm{~mm}$ y $6 \mathrm{~cm}$ de arena de $2 \mathrm{~mm}$. Además, se colocó una malla a la salida del tanque para evitar pérdida de material granular. La altura de $10 \mathrm{~cm}$ de arena de $0,5 \mathrm{~mm}$ se definió considerando la capacidad del tanque basado en el rango de valores de $20 \mathrm{~cm}$ (Ali et al., 2001) a 7,62 cm (Meng, Korfiatis, Christodoulatos y Bang, 2001) reportados en la literatura para sistemas de punto de uso. Se 
utilizó arena como un material reutilizable, que se extrae del filtro y se lava con agua, como se ha hecho con otros sistemas de punto de uso (Ali et al., 2001; Meng et al., 2001). Inicialmente, ambos tanques se conectaron a través de la tubería de conexión por gravedad, sin embargo, en la práctica se determinó que es suficiente usar un pascón de cocina como difusor para distribuir el agua en la superficie del medio filtrante. Por último, se colocó otra llave que se ubica en la parte inferior del filtro para la salida del agua. Para proporcionar la agitación necesaria para la coagulación y floculación se utilizó un molinillo de madera.

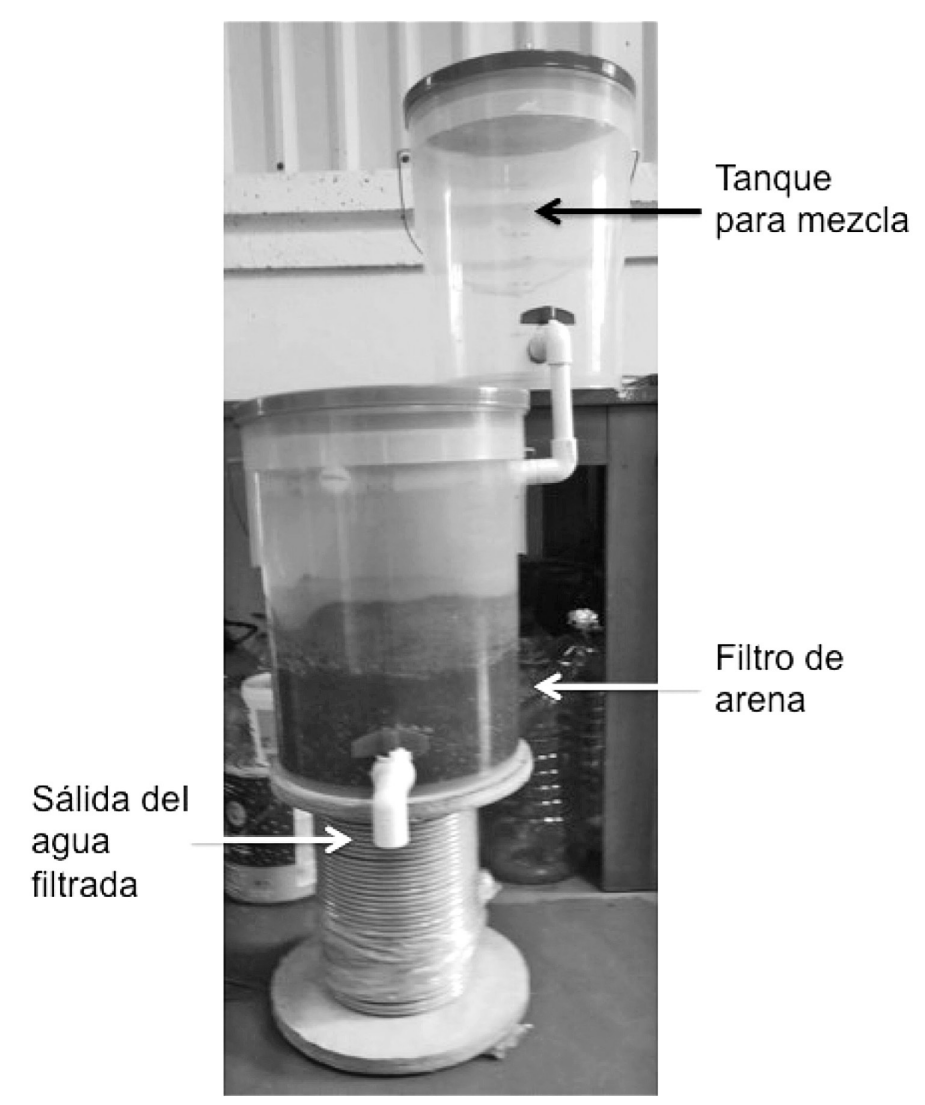

Figura 1. Prototipo desarrollado para la remoción domiciliar de arsénico

\section{Pruebas al prototipo para remoción de arsénico de uso doméstico}

Después de la confección del prototipo se realizaron 10 pruebas con $15 \mathrm{~L}$ del agua NSF y con las condiciones óptimas encontradas anteriormente. También, se evaluaron, utilizando las mismas condiciones, una muestra de agua de la Zona Norte y otra del Pacífico Norte del país con el fin de probar la eficiencia del prototipo con agua de las zonas afectadas. La concentración de arsénico de la muestra de la Zona Norte fue de $50 \mu \mathrm{g} / \mathrm{L}$ y de la muestra del Pacífico Norte de $10 \mu \mathrm{g} / \mathrm{L}$. Estas muestras se evaluaron de forma demostrativa a personas de las comunidades afectadas durante giras de campo.

\section{Métodos de análisis y composición del agua reto}

La determinación del contenido de arsénico en agua se realizó mediante el método 3114b del Standard Methods (APHA, AWWA y WPCF, 2005) por espectrofotometría de absorción atómica (AA) por generación de hidruros (Clesceri, Greenberg y Eaton, 1999). Se utilizó un 
espectrofotometro marca Perkin Elmer AAnalyst 800. El método consistió en la generación de hidruros y la formación de $\mathrm{AsH}_{3}$ (arsina) mediante la reacción con el borohidruro de sodio y una mezcla reductora (mezcla 1:1 de disoluciones acuosas de yoduro de potasio al $5 \% \mathrm{~m} / \mathrm{v}$ y ácido ascórbico al $5 \% \mathrm{~m} / \mathrm{v}$ ). Posteriormente, el arsénico trivalente total se analizó en el espectrómetro de absorción atómica, en el cual se convirtieron en átomos en su fase gaseosa (Armienta, Castro de Esparza, López, Piñeiro y Sassone, 2009). El límite de detección es de 0,03 ㅆg/L y el de cuantificación de 0,1 $\mu \mathrm{g} / \mathrm{L}$. Estos se determinaron según lo que indican Miller y Miller (2002). Por otro lado, la incertidumbre de $1 \mu \mathrm{g} / \mathrm{L}$ se calculó según el procedimiento descrito por Rivas y Fernández (2006).

Cuadro 1. Compocisión del agua tipo reto (Amy et al., 2005)

\begin{tabular}{|c|c|}
\hline Parámetro & Concentración $(\mathrm{mg} / \mathrm{L})$ \\
\hline Sodio & 74 \\
\hline Calcio & 40 \\
\hline Magnesio & 13 \\
\hline Alcalinidad $\left(\mathrm{CaCO}_{3}\right)$ & 150 \\
\hline Cloruros & 71 \\
\hline Fluoruros & 1 \\
\hline Sulfatos & 48 \\
\hline Nitratos $(\mathrm{N})$ & 2 \\
\hline Fosfato $(\mathrm{P})$ & 0,12 \\
\hline Sílice $(\mathrm{SiO}$ & \\
\hline
\end{tabular}

Fuente: Amy et al., 2005.

Para el análisis de hierro residual se siguió el método de fenantrolina 3500-Fe B del Standard Methods (APHA et al., 2005) utilizando un colorímetro LaMotte modelo Smart 3. El límite de detección fue de $0,12 \mathrm{mg} / \mathrm{L}$. Se utilizó el mismo equipo en la determinación de turbidez por el método de absorción. Además, los métodos de muestreo, preservación y manipulación de las muestras se realizaron siguiendo las recomendaciones del Standard Methods (APHA et al., 2005). El agua tipo reto consistió en la adición de una serie de sales tipo primario a agua ultra pura (Mille Q) según el cuadro 1.

\section{Resultados y discusión}

\section{Remoción de arsénico mediante el sistema SORAS modificado}

Según Litter, Morgada y Bundschuh (2010), la oxidación de hierro cero-valente en la presencia de agua y oxígeno produce hierro (II) y posteriormente diferentes óxidos o hidróxidos de hierro (II) y (III). En dichos materiales el arsénico puede adsorberse o coprecipitar. La figura 2 muestra que la remoción del arsénico hasta niveles por debajo de $10 \mu \mathrm{g} / \mathrm{L}$ ocurrió después de dos horas de exposición en el caso de días soleados (líneas B y C) y cuatro horas cuando el día estuvo nublado (línea A). El tiempo de exposición necesario para la remoción por debajo del límite establecido en los experimentos realizados en días soleados resultó igual al reportado por Litter 
y colaboradores (2012) utilizando la misma dosificación de hierro y agua sintética pero con luz ultravioleta artificial (366 $\left.\mathrm{nm} ; 0,800 \mathrm{~W} / \mathrm{m}^{2}\right)$.

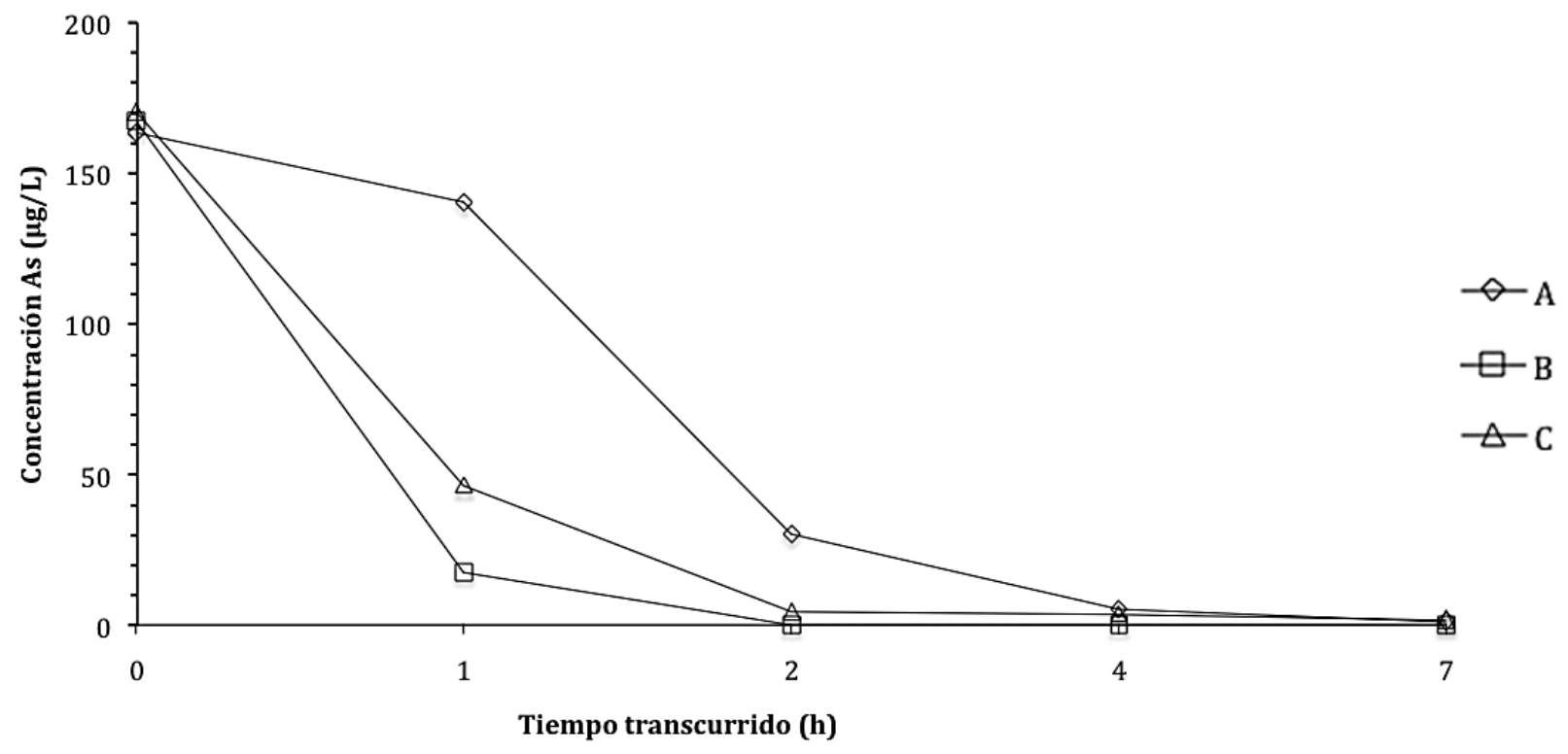

Figura 2. Remoción de arsénico mediante SORAS modificado

Como ilustra la figura $3 a$, en las condiciones en que se realizó el presente estudio para el caso del experimento $A$, al estar el día nublado, no se tuvo suficiente radiación durante todo el tiempo de exposición, siendo menor a $0,400 \mathrm{~W} / \mathrm{m}^{2}$ después de la primera hora y media. En el caso del segundo experimento, como se muestra en la figura 3b, se consideró como un día bastante soleado (la radiación siempre estuvo por encima de 1,200 W/m² durante las primeras dos horas de exposición). Finalmente, en la figura 3c se indica que la radiación estuvo por encima de $0,600 \mathrm{~W} / \mathrm{m}^{2}$ logrando una remoción eficiente de arsénico después de dos horas. Así, los datos sugieren que probablemente la radiación minima durante dos horas está cerca de 0,600 W/ $\mathrm{m}^{2}$. No obstante, en el caso de un día nublado se debe extender el tiempo de exposición. Se requieren investigaciones adicionales para determinar estos límites de exposición y características del día.

Por último, el agua obtenida después del proceso de filtración presentó en algunos casos concentraciones de hierro levemente superiores a la norma $(0,3 \mathrm{mg} / \mathrm{L})$, lo que sugiere que se deben realizar más experimentos para definir esta variable. Esta variable, al igual que la influencia del $\mathrm{pH}$ del agua que va a ser tratada y el trabajo con aguas reales, son aspectos que se están investigando. 

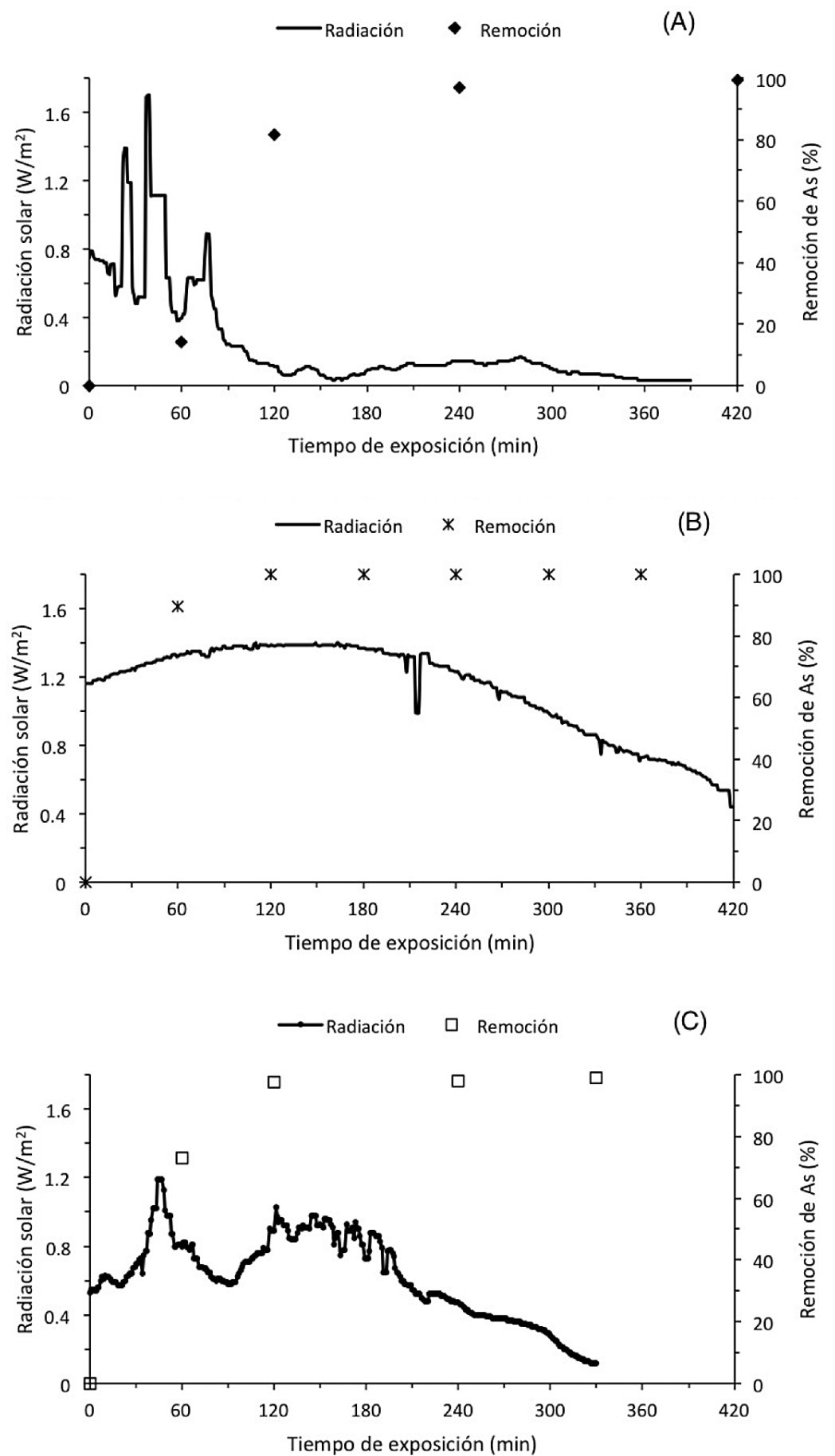

Figura 3. Remoción de arsénico por el método de SORAS modificado en tres diferentes condiciones de radiación natural

\section{Condiciones apropiadas de floculación-coagulación}

La efectividad de un proceso de coagulación-floculación depende de una serie de variables que se deben optimizar. En el cuadro 2 se observan las condiciones determinadas mediante pruebas de jarras para la remoción efectiva de arsénico de agua reto NSF hasta valores por debajo de la normativa. El detalle de los ensayos realizados para obtener dichas condiciones lo describe Rojas-Chaves (2014). 
Tecnología en Marcha,

62 Vol. 28, N. ${ }^{\circ} 4$, Octubre-Diciembre 2015

Cuadro 2. Condiciones óptimas de remoción de arsénico por coagulación/floculación usando cloruro de hierro y dos diferentes floculantes

\begin{tabular}{|c|c|c|}
\hline Parámetro & Polimero catiónico & Mozote \\
\hline $\mathrm{pH}$ & 6 & 14 \\
\hline $\mathrm{FeCl}_{3}(\mathrm{mg} / \mathrm{L})$ & 12 & 250 \\
\hline Floculante $(\mathrm{mg} / \mathrm{L})$ & 1 & 1 \\
\hline Tiempo de floculación $(\mathrm{min})$ & 1 & 20 \\
\hline Tiempo de sedimentación $(\mathrm{min})$ & 20 & 6 \\
\hline
\end{tabular}

En relación con el pH, de manera similar a lo reportado por Lakshmanan, Clifford y Samanta (2008), la eficiencia del tratamiento aumentó conforme el pH del agua disminuyó de 7,25 a 6,00, siendo este último el valor óptimo para la remoción del arsénico sobre la superficie del hidróxido de hierro formado en el agua. Además, la concentración de cloruro de hierro (III) óptima es baja en comparación con otros sistemas de punto de uso, en los que las concentraciones de cloruro de hierro (III) fueron de 20 mg/L (Ali et al., 2001) y 25 mg/L (Bordoloi, Nath, Gogoi y Dutta, 2013). En este caso, se obtuvieron mejores resultados debido a que, contrariamente a los estudios citados, se usó un floculante y para el caso de Ali y colaboradores (2001) y se utilizaron concentraciones más altas de arsénico en el orden de 500-1000 $\mu \mathrm{g} / \mathrm{L}$. El aumento en la remoción de arsénico al utilizar un floculante de tipo catiónico también ha sido observado por Cheng y colaboradores (1994) cuando la concentración de cloruro de hierro (III) es menor o igual a $20 \mathrm{mg} / \mathrm{L}$.

La concentración óptima de polímero catiónico estuvo en el rango de 1-1,8mg/L con una remoción del 96\%. En el caso del mozote, la dosis óptima fue de $250 \mathrm{mg} / \mathrm{L}$ correspondiente a una remoción del 98\%. Esta dosis se encuentra dentro del rango (200-400 mg/L) similar a la obtenida por Quirós y colaboradores (2010b) al aplicar mozote en la remoción de 100\% de turbiedad y color en agua sintética con 20 UNT de turbiedad inicial. Es importante señalar que en el estudio mencionado se empleó sulfato de aluminio como coagulante, con una dosis de 1 $\mathrm{mg} / \mathrm{L}$.

Considerando que la intención es utilizar el sistema estudiado a nivel domiciliar, una agitación manual prolongada puede resultar difícil para el usuario, por lo que se evaluó el tiempo de floculación. Los porcentajes de remoción de arsénico fueron mayores al 90\% en todos los tiempos empleados. Para el floculante natural, se tomó como tiempo óptimo 1 minuto, cuya concentración final de arsénico correspondió a $4 \mu \mathrm{g} / \mathrm{L}$. Por otro lado, a pesar de que la mayor remoción con el polímero catiónico fue a los 4 minutos, en 1 minuto se alcanzó una concentración final de arsénico de $9 \mu \mathrm{g} / \mathrm{L}$, por lo que se seleccionó como tiempo óptimo.

Otro aspecto importante que se logró determinar fue el tiempo mínimo de sedimentación necesario para evitar la rápida saturación del filtro de arena. Así, se definió el tiempo requerido para obtener una turbiedad de 5 UNT (valor máximo admisible por el Reglamento de Calidad de Agua Potable (Ministerio de Salud, 2005) previo a la filtración. Al utilizar el polímero, a partir de los 23 minutos la turbiedad alcanzó el valor mencionado, mientras que con el mozote la sedimentación fue un poco más rápida, a los 20 minutos, en que alcanzó un valor menor de 5 UNT. El anterior valor de tiempo se seleccionó para mayor facilidad tanto para el polímero catiónico como para el mozote. 


\section{Pruebas al prototipo para remoción de arsénico de uso doméstico}

Una vez obtenidas las condiciones óptimas de coagulación-floculación, se realizaron los ensayos utilizando el prototipo de uso domiciliar (figura 1). Al utilizar el agua tipo NSF con $200 \mathrm{\mu gAs} / \mathrm{L}$ en 10 ensayos diferentes, el agua tratada presentó concentraciones de arsénico residual inferior a los $10 \mu \mathrm{g} / \mathrm{L}$ (remoción del 99\%). Con respecto a otros sistemas de punto de uso similares, el prototipo del presente estudio empleó una cantidad de cloruro de hierro (III) inferior. En el caso de los sistema de Ali y colaboradores (2001) y Bordoloi y colaboradores (2013), las dosis fueron de 20 y 25 mg/L de $\mathrm{FeCl}_{3}$ y el rango de arsénico entre 500-1000 y 196$212 \mu \mathrm{g} / \mathrm{L}$, respectivamente. Dos aspectos importantes que se controlaron fueron la cantidad de hierro residual y la turbiedad residual después de realizada la filtración, ya que podían impartirle sabor, color y material particulado al agua, respectivamente. En los ensayos realizados, solo en la primera prueba la turbiedad estuvo en 6,0 \pm 01 UNT, levemente por encima de la norma (5,0 UNT).

Al evaluar el sistema utilizando dos muestras de agua de las zonas efectadas (con 50 $\mathrm{HgAs} / \mathrm{L}$ y $10 \mu / L)$, el porcentaje de remoción en estos casos fue de $90 \%$ y $70 \%$, respectivamente. Ambos casos menores que el resultante con las pruebas con el agua NSF; sin embargo, la concentración de arsénico cumplió la normativa. De igual manera, la turbiedad, el color y el hierro residual estuvieron por debajo de lo requerido. Además, las muestras filtradas no presentaron problemas de olor o sabor. Entre las razones por las que se obtuvieron eficiencias ligeramente menores a las encontradas con el agua NSF, es importante destacar que no se conocía la especiación de arsénico en el agua; este factor puede haber influido debido a que una mayor proporción de arsénico trivalente disminuye la eficiencia del tratamiento, dado que es más dificil de remover del agua que el arsénico pentavalente (Áviles, Tirado, Bedolla y Ramírez, 2003; Sancha, 2010); lo cual es muy probable, ya que al tratarse de agua subterránea, posiblemente predomina el arsénico trivalente (Herrera, 2013). Otra razón que puede reducir el porcentaje de remoción es la presencia de interferencias en el agua, como el sílice y fosfatos (Lakshmanan, 2007; Laky y Licskó, 2011). Según Araya (com. Pers., diciembre de 2014) en las zonas afectadas se han encontrado concentraciones de sílice de hasta $120 \mu \mathrm{g} / \mathrm{L}$, muy superiores a $20 \mathrm{mg} / \mathrm{L}$ del agua NSF.

\section{Conclusiones y recomendaciones}

El uso del procedimiento SORAS modificado durante días soleados con suficiente radiación natural (posiblemente por encima de 0,600 W/m²) permite la remoción de arsénico desde 200 $\mu \mathrm{g} / \mathrm{L}$ hasta valores por debajo de la norma en al menos 2 horas de exposición. En días nublados se debe extender ese tiempo hasta las 4 horas. No obstante, se requiere más investigación para definir estos tiempos adecuadamente. Aunado a ello, el agua producida por el método SORAS modificado puede presentar residuales de hierro por encima de la norma (0,3 mg/L), por tanto, se debe determinar la cantidad mínima de lana de hierro que permita una remoción apropiada de arsénico y un residual de hierro seguro.

En el caso de la técnica de coagulación-floculación, el valor óptimo de pH fue el de 6, lo cual es congruente con lo expuesto en la literatura, que indica que a $\mathrm{pH}$ menor de 8 , el porcentaje de remoción es mayor. Las dosis de cloruro de hierro (III) de 12 y 14 mg/L para el polímero y el mozote, respectivamente, son bajas en comparación con otros sistemas de punto de uso que han empleado concentraciones de entre 20 y $25 \mathrm{mg} / \mathrm{L}$. Estas bajas dosis de coagulante derivan en un tratamiento menos costoso. Las concentraciones óptimas de los floculantes fueron de $1 \mathrm{mg} / \mathrm{L}$ de polímero y $250 \mathrm{mg} / \mathrm{L}$ de mozote. En el caso del polímero catiónico, es una dosis baja pues se han utilizado concentraciones de hasta $4 \mathrm{mg} / \mathrm{L}$. Por otro lado, el mozote no se ha 
usado para la remoción de arsénico, sin embargo, el valor utilizado es bajo, ya que las dosis empleadas para la eliminación de turbiedad se encuentran entre 200 y 400 mg/L.

Entre las principales ventajas que presenta el prototipo está el hecho de que es desmontable, lo que facilita su mantenimiento; además, su confección es muy sencilla. Otra ventaja es su bajo costo, no solo en los materiales necesarios sino también en los reactivos utilizados en el tratamiento, como el cloruro de hierro (III) y el mozote. El prototipo presentó una menor eficiencia con las aguas naturales; esto se debe probablemente a que puede existir arsénico trivalente o la presencia de otras interferencias en el agua, como el sílice. Sin embargo, con el tratamiento se obtuvo una concentración de arsénico menor a $10 \mu \mathrm{g} / \mathrm{L}$, lo cual es de esperarse puesto que al emplear el agua NSF con la intención de probar el sistema, se vaticina que sea eficaz en la remoción de arsénico. Además, es efectivo en la remoción del hidróxido de hierro (III) del agua, lo que se refleja en la calidad del agua en los valores de hierro total, turbiedad y color.

Finalmente, aunque en el agua reto se incluyen especies interferentes, en ambas tecnologías se debe evaluar su efecto con más detalle. Dado que cada tipo de agua presenta características únicas, antes de aplicar la tecnología en un agua específica se recomienda efectuar los ensayos pertinentes que garanticen una remoción de arsénico apropiada.

\section{Bibliografía}

Ali, M.A., Badruzzaman, A.B.M., Jalil, M.A., Hossain, M.D., Hussainuzzaman, M.M., Badruzzaman, M. \& Akter, N. (2001). Development of low-cost technologies for removal of arsenic from groundwater. En Bangladesh University of Engineering \& Technology \& United Nations University (Eds.), Technologies for Arsenic Removal from Drinking Water (pp. 99-120). Dhaka.

Amy, G., Chen, H.C., Drizo, A., von Gunten, U., Brandhuber, P., Hund, R. \& Banerjee, K. (2005). Adsorbent treatment technologies for arsenic removal (p. 164). Denver.

APHA, AWWA \& WPCF. (2005). Standard methods for the examination of water and wastewater. 21 ed. Washington D.C.: American Public Health Association.

Armienta, M.A., Castro de Esparza, M.L., López, C.M., Piñeiro, A.E. \& Sassone, A.H. (2009). Determinación de arsénico por el método: absorción atómica-generación de hidruros-FIAS. En M.I. Litter, M.A. Armienta y S. Farías (Eds.), Metodologías analíticas para la determinación y especiación de arsénico en aguas y suelos. Argentina: CYTED.

Astorga, Y. (2013). Decinoveno Informe Estado de la Nación en Desarrollo Humano Sostenible: Gestión del recurso hídrico. San José: Programa Estado de la Nación.

Avilés, M., Tirado, M., Bedolla, L. \& Ramírez, A. (2003). Remoción de arsénico a nivel domiciliar. En C. Díaz (Ed.), Agua potable para comunidades rurales, reúso y tratamientos avanzados de aguas residuales domésticas (pp. 1-14). Madrid: Red Iberoamericana de Potabilización y Depuración del Agua.

Bordoloi, S., Nath, S.K., Gogoi, S. \& Dutta, R.K. (2013). Arsenic and iron removal from groundwater by oxidationcoagulation at optimized $\mathrm{pH}$ : laboratory and field studies. Journal of Hazardous Materials, 260, 618-26. doi:10.1016/j.jhazmat.2013.06.017

Bundschuh, J., Litter, M.I., Parvez, F., Román-Ross, G., Nicolli, H.B., Jean, J.S. \& Toujaguez, R. (2012). One century of arsenic exposure in Latin America: A review of history and occurrence from 14 countries. Science of the Total Environment. doi:10.1016/j.scitotenv.2011.06.024

Clesceri, L., Greenberg, A. \& Eaton, A. (Eds.). (1999). Standard Methods for the Examination of Water and Wastewater. 20 ed. American Public Health Association, American Water Works Association, Water Environment Federation.

Herrera, L.M. (mayo, 2013). Contaminación con arsénico afecta agua de 24 pueblos. La Nación.

Hwang, S.K. (2002). Point-of-Use Arsenic Removal from Drinking Water in Nepal Using Coagulation and Filtration. Massachusetts Institute Of Technology.

Jain, C.K. \& Singh, R.D. (2012). Technological options for the removal of arsenic with special reference to South East Asia. Journal of Environmental Management, 107, 1-18. doi:10.1016/j.jenvman.2012.04.016 
Lakshmanan, D. (2007). A systematic study of arsenic removal from drinking water using coagulation-filtration and electrocoagulation-filtration. University of Houston.

Lakshmanan, D., Clifford, D. \& Samanta, G. (2008). Arsenic Removal by Coagulation with Aluminium, Iron, Titanium, and Zirconium. American Water Works Association, 100(2), 76-88.

Laky, D. \& Licskó, I. (2011). Arsenic removal by ferric-chloride coagulation - effect of phosphate, bicarbonate and silicate. Water Science \& Technology, 64(5), 1046. doi:10.2166/wst.2011.419

Litter, M.I., Morgada, M.E., Lin, H., García, M.G., Hidalgo, M. del V., D'Hiriart, J. \& Mateu, M. (2010). Experiencias de remoción de arsénico por tecnologías fotoquímicas y solares en Argentina. En M.I. Litter, A.M. Sancha y A.M. Ingallinella (Eds.), Iberoarsen Tecnologías económicas para el abatimiento del arsénico en aguas (pp. 191208). Argentina: CYTED.

Litter, M.I., Alarcón-Herrera, M.T., Arenas, M.J., Armienta, M.A., Avilés, M., Cáceres, R.E. \& Pérez-Carrera, A. (2012). Small-scale and household methods to remove arsenic from water for drinking purposes in Latin America. The Science of the Total Environment, 429, 107-22. doi:10.1016/j.scitotenv.2011.05.004

Meng, X., Korfiatis, G.P., Christodoulatos, C. \& Bang, S. (2001). Treatment of arsenic in Bangladesh well water using a household co-precipitation and filtration system. Water Research, 35(12), 2805-2810. doi:10.1016/S00431354(01)00007-0

Miller, J.N. \& Miller, J. (2002). Métodos de calibración en análisis instrumental: regresión y correlación. En Estadística y Quimiometría para Química Analítica. 4 ed. (pp. 111-155). Madrid: Pearson Educación, S.A.

Ministerio de Salud. (2005). Reglamento para la Calidad del Agua Potable. Pub. L. No. 32327-S (2005). Costa Rica: Diario La Gaceta No. 84.

OPS. (2005). Guía para el mejoramiento de la calidad del agua a nivel casero. Lima: OPS.

Programa Estado de la Nación. (2013). Decimonoveno Informe Estado de la Nación en Desarrollo Humano Sostenible. San José: PEN.

Quirós, N., Vargas, M. \& Jiménez, J. (2010). Extracción y análisis de polímeros obtenidos a partir de varios productos naturales, para ser usados como potenciales floculantes en el tratamiento de agua para consumo humano (pp. 1-30). Cartago, C.R.: Instituto Tecnológico de Costa Rica.

Rivas, H. \& Fernández, P. (2006). Estimación de incertidumbre para medición de Zn por espectrofotometría de absorción atómica-flama. En Simposio de Metrología (p. 6).

Rojas-Chaves, P. (2014). Diseño de un prototipo de uso doméstico para la remoción de arsénico en agua potable, basado en coagulación/floculación con cloruro de hierro (III). Cartago, C.R.: Instituto Tecnológico de Costa Rica.

Sancha, A.M. (2010). Remoción de arsénico por coagulación y precipitación. En M.I. Litter, A.M. Sancha y A.M. Ingallinella (Eds.), Tecnologias económicas para el abatimiento de arsénico en aguas (pp. 33-41). Argentina: CYTED.

WHO. (2011). Guidelines for Drinking-water Qualit. 4 ed. Ginebra: World Health Organization. 